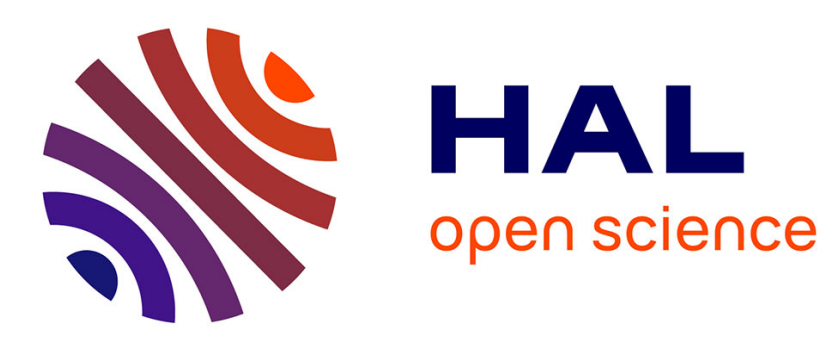

\title{
Benchmarking of wildland fire color segmentation algorithms
}

\author{
T Toulouse, Lucile Rossi, M Akhloufi, T Celik, Xavier Maldague
}

\section{To cite this version:}

T Toulouse, Lucile Rossi, M Akhloufi, T Celik, Xavier Maldague. Benchmarking of wildland fire color segmentation algorithms. IET Image Processing, 2015, 9 (12), pp.1064-1072. 10.1049/ietipr.2014.0935 . hal-01182107

\section{HAL Id: hal-01182107 https://hal.science/hal-01182107}

Submitted on 7 Nov 2017

HAL is a multi-disciplinary open access archive for the deposit and dissemination of scientific research documents, whether they are published or not. The documents may come from teaching and research institutions in France or abroad, or from public or private research centers.
L'archive ouverte pluridisciplinaire HAL, est destinée au dépôt et à la diffusion de documents scientifiques de niveau recherche, publiés ou non, émanant des établissements d'enseignement et de recherche français ou étrangers, des laboratoires publics ou privés. 


\title{
Benchmarking of wildland fire color segmentation algorithms
}

\author{
T. Toulouse ${ }^{a, b}$, L. Rossi ${ }^{a}$, M. Akhloufi ${ }^{b, c}$, T. Celik $^{d}$, X. Maldague ${ }^{b}$ \\ ${ }^{a}$ UMR CNRS 6134 SPE, University of Corsica, 20250 Corte, France \\ ${ }^{b}$ Electrical and Computer Engineering, Laval University, Quebec, QC, G1V0A6 Canada \\ ${ }^{c}$ Department of Electronics Engineering, Universidad Tecnica Federico Santa Maria, \\ Valparaso, Chile \\ ${ }^{d}$ School of Computer Science, University of the Witwatersrand, \\ Johannesburg, South Africa \\ \{lrossi, toulouse\}@univ-corse.fr
}

This paper is a postprint of a paper submitted to and accepted for publication in IET Image Processing (doi: 10.1049/iet-ipr.2014.0935) and is subject to Institution of Engineering and Technology Copyright. The copy of record is available at IET Digital Library.

\begin{abstract}
Recently, computer vision-based methods have started to replace conventional sensor based fire detection technologies. In general, visible band image sequences are used to automatically detect suspicious fire events in indoor or outdoor environments. There are several methods which aim to achieve automatic fire detection on visible band images, however, it is difficult to identify which method is the best performing as there is no fire image dataset which can be used to test the different methods. This paper presents a benchmarking of state of the art wildland fire color segmentation algorithms using a new
\end{abstract}


fire dataset introduced for the first time in this paper. The dataset contains images of wildland fire in different contexts (fuel, background, luminosity, smoke). All images of the dataset are characterized according to the principal color of the fire, the luminosity and the presence of smoke in the fire area. With this characterization, it has been possible to determine on which kind of images each algorithm is efficient. Also a new probabilistic fire segmentation algorithm is introduced and compared to the other techniques. Benchmarking is performed in order to assess performances of twelve algorithms that can be used for the segmentation of wildland fire images.

\section{Introduction}

In Europe, up to 10,000 $\mathrm{km}^{2}$ of vegetation are burned by fire each year. In North America and Russia it is up to $100,000 \mathrm{~km}^{2}$ [1]. More recently new events provide examples of disasters caused by fires: in June 2013 in Arizona (United States of America), 19 firefighters were killed and 100 houses were burned by a wildfire. In August 2013, a forest fire in the state of California caused significant damage to more than 111 structures and has burned 1,042 km² of land. The cost of fire fighting was estimated to $\$ 127.35$ million [1].

Forest fires are a major risk in many countries around the world. They cause environmental damage, deforestation, desertification, air pollution, $\left(\mathrm{CO}_{2}\right.$ emissions into the atmosphere which represent $20 \%$ of total emissions, recirculation of heavy metals and radionuclides), economic losses (destruction of housing and production facilities including forest fighter material losses) and loss of lives.

The fight against this major risk is more effective when it is possible to detect its early departure and to anticipate the fire behavior over time and especially its position and its intensity. In order to develop fire behavior models and to improve them, it is necessary to have experimental data that characterize the fire propagation $[2,3,4]$. Information like the position, rate of spread, height, inclination, surface and volume are important characteristics 
[5]. In recent years, we assist to an increasing interest to develop remote techniques based on computer vision to detect and characterize wildland fires.

The first and most important step for computer vision processing is the image segmentation process because it determines the accuracy of the following steps. The wildland fires segmentation is non trivial, for example we can see the following problems: the background is different for each fire and can be complex, the luminosity can change during a fire propagation, the color of the fire varies from yellow to red and smoke with different degree of transparency can be present.

In the area of fire detection, different algorithms were proposed in order to detect fire in images or video sequences [6]; the result of these detections are a decision about the presence of the fire in the image. These algorithms are not suitable for a precise segmentation of the fire. In a metrology purpose, it is important to find the most effective and robust wildland fire segmentation algorithm. Fire segmentation algorithms use color criteria in different color spaces: $R G B[7,8,9,10,11], Y C b C r[12,13], Y U V[14], L^{*} a^{*} b^{*}[15], H S I$ [16], HSV [17] or combination of different color spaces $[18,19]$. In the case of fire detection in video sequences, some methods use background extraction [12], flicker analysis [8], or shape analysis [17]. In this paper, we emphasize on color segmentation methods often used as a first step in the fire segmentation process. In this work, eleven state of the art color segmentation methods $[7,9,10,11,12,13,15,16,18,19,20]$ used to extract wildfire regions has been selected. Also, a new color segmentation algorithm is proposed and compared with the above algorithms. Past works were proposed in order to evaluate the performance of some algorithms $[11,13,21,22]$. These works were conducted using different datasets, and did not take into account fire characterization as proposed in our work. In this paper, the performances of the selected segmentation algorithms on a characterized dataset containing one hundred wildland fire images are presented. Main contributions of this paper are: a new wildland fire dataset 
with various contexts and its fire segmentation ground truth, a categorization of this dataset using different characteristics (smoke, color, etc.), a new probabilistic approach for wildland fire segmentation using color vision, a benchmarking of the state of the art fire segmentation methods present in the literature, and a benchmarking website for testing new developed fire segmentation algorithms.

The paper is organized as follow: Section 2 presents the color segmentation algorithms, section 3 describes the four criteria for benchmarking, section 4 presents the image dataset and the image characterization methods, section 5 presents the evaluation results and comparison of the wildland fire segmentation methods, finally, section 6 gives the conclusions.

\section{Fire segmentation methods}

In this section, twelve methods of fire color segmentation in the visible spectrum are introduced. In order to automatically detect fire in visible band images, different color spaces have been used as can be seen in Table 1. In this section, the methods are presented according to the working color space of the segmentation. In the following, $\boldsymbol{I}$ represents an input image, $\boldsymbol{I}(\mathbf{x})$ is the image pixel localized at spatial location $(\mathbf{x})$ and $\boldsymbol{I}_{c}(\mathbf{x})$ represents the pixel of channel $c$ of the corresponding color space.

Table 1: Color spaces of the 12 compared segmentations

\begin{tabular}{lccccc}
\hline & $R G B$ & $Y C_{b} C_{r}$ & $H S I$ & $Y U V$ & $L^{*} a^{*} b^{*}$ \\
\hline Phillips et al. 2002 [7] & $\sqrt{ }$ & & & & \\
Chen et al. 2004 [18] & $\sqrt{ }$ & & $\sqrt{ }$ & & \\
Horng et al. 2005 [16] & $\sqrt{ }$ & & & & \\
Celik et al. 2007 [9] & & $\sqrt{ }$ & & & \\
Celik and Demirel 2009 [12] & $\sqrt{ }$ & & & $\sqrt{ }$ & \\
Ko et al. 2009 [10] & $\sqrt{ }$ & & & & $\sqrt{ }$ \\
Rossi and Akhlouf 2010 [19] & & & & & $\sqrt{ }$ \\
Chitade and Katiyar 2010 [20] & $\sqrt{ }$ & & & & \\
Celik 2010 [15] & $\sqrt{ }$ & $\sqrt{ }$ & & & \\
Collumeau et al. 2011 [11] & & & \\
Rudz et al. 2013 [13] & & & \\
The proposed bayesian fire detection & & $\sqrt{ }$ & & \\
\hline
\end{tabular}




\section{1 $R G B$ color space segmentation}

Phillips et al. [7] use a training dataset composed of manually labeled data. A look up table is created from a 3D Gaussian smoothing histogram $(256 \times 256 \times 256)$ of the training images. The Gaussian smoothing histogram is computed for the $R G B$ color space as follows: for each fire pixel of the image, a 3D Gaussian distribution with a standard deviation $\sigma=2$ is added to the color histogram centered in the pixel $(R, G, B)$ value and for each background pixel a 3D Gaussian distribution with $\sigma=1$ is subtracted from the color histogram. The Gaussian smoothing histogram is computed for each image of the training dataset, and the look up table is created from these histograms. Then a threshold is chosen to classify a pixel into fire pixel or non-fire pixel according to the value of the look up table on the coordinate corresponding to the $(R, G, B)$ pixel value.

Celik et al. [9] use 150 fire images collected from Internet to generate a set of rules in $R G B$ color space. A pixel ( $\mathbf{x})$ is considered as a fire pixel if the following conditions are satisfied:

$$
\begin{aligned}
& \boldsymbol{I}_{R}(\mathbf{x})>R_{\text {Mean }} ; \\
& \boldsymbol{I}_{R}(\mathbf{x})>\boldsymbol{I}_{G}(\mathbf{x})>\boldsymbol{I}_{B}(\mathbf{x}) \\
& 0.25 \leq \frac{\boldsymbol{I}_{G}(\mathbf{x})}{\boldsymbol{I}_{R}(\mathbf{x})+1} \leq 0.65 \\
& 0.05 \leq \frac{\boldsymbol{I}_{B}(\mathbf{x})}{\boldsymbol{I}_{R}(\mathbf{x})+1} \leq 0.45 \\
& 0.20 \leq \frac{\boldsymbol{I}_{B}(\mathbf{x})}{\boldsymbol{I}_{G}(\mathbf{x})+1} \leq 0.60
\end{aligned}
$$

where $R_{\text {Mean }}$ is the mean of the red channel values of all the image pixels: $R_{\text {Mean }}=$ $\frac{1}{N} \sum_{\mathbf{x} \in \boldsymbol{I}} \boldsymbol{I}_{R}(\mathbf{x})$, with $N$ the number of pixels in the image.

Ko et al. [10] propose the use of a Gaussian probability distribution of each channel in $R G B$ color space: 


$$
p_{c}\left(\boldsymbol{I}_{c}(\mathbf{x})\right)=\frac{1}{\sqrt{2 \pi \sigma_{c}^{2}}} \exp \left(-\frac{\left(\boldsymbol{I}_{c}(\mathbf{x})-\mu_{c}\right)^{2}}{2 \sigma_{c}^{2}}\right)
$$

where $\mu_{c}$ and $\sigma_{c}$ are respectively the mean and the standard deviation of channel $c \in$ $\{R, G, B\}$ of fire pixels. In [10], these parameters are computed using 100 images of dynamic fires captured from 10 video sequences. Using Eq. (2), the total probability that a pixel (x) is a fire pixel is computed as the product of the probability $p_{c}$ of each $R G B$ channel:

$$
p_{f}(\mathbf{x})=\prod_{c \in\{R, G, B\}} p_{c}\left(\boldsymbol{I}_{c}(\mathbf{x})\right)
$$

Using the above equation, a pixel $(\mathbf{x})$ is classified as fire if $p_{f}(\mathbf{x}) \geq \tau$, where $\tau$ is a threshold value. In [10], there is no information about how the value of $\tau$ is selected. The value used in this paper and its computation is specified in section 5

A method using Support Vector Machines (SVM) classifier on the $R G B$ color space has been developed by Collumeau et al. [11]. The authors use a set of 12000 training pixels, labeled as fire or non-fire. Scattered false positives are removed with a morphological opening operator, applied to the SVM outputs. Then, in order to remove bright false positive pixels, the intensity of segmented regions is analyzed. The mean of the intensity is computed for these regions. In the regions where the mean of intensity is upper than a threshold $\tau$, pixels greater than the same threshold $\tau$ are removed from the segmentation. A threshold of $80 \%$ is used by the authors.

\section{$2.2 Y C_{b} C_{r}$ color space segmentation}

A set of rules in the $Y C_{b} C_{r}$ color space is defined for a method proposed by Celik and Demirel [12] in order to use the consistency in chromaticity representation. A pixel (x) must satisfy 
the following rules to be classified as a fire pixel:

$$
\begin{aligned}
& \boldsymbol{I}_{Y}(\mathbf{x})>\boldsymbol{I}_{C b}(\mathbf{x}) ; \\
& \boldsymbol{I}_{C r}(\mathbf{x})>\boldsymbol{I}_{C b}(\mathbf{x}) ; \\
& \boldsymbol{I}_{Y}(\mathbf{x})>Y_{\text {Mean }} ; \\
& \boldsymbol{I}_{C b}(\mathbf{x})<C b_{\text {Mean }} ; \\
& \boldsymbol{I}_{C r}(\mathbf{x})>C r_{\text {Mean }} ; \\
& \left|\boldsymbol{I}_{C b}(\mathbf{x})-\boldsymbol{I}_{C r}(\mathbf{x})\right| \geq \tau ; \\
& \boldsymbol{I}_{C b}(\mathbf{x}) \geq f_{u}\left(\boldsymbol{I}_{C r}(\mathbf{x})\right) ; \\
& \boldsymbol{I}_{C b}(\mathbf{x}) \leq f_{l}\left(\boldsymbol{I}_{C r}(\mathbf{x})\right) ; \\
& \boldsymbol{I}_{C b}(\mathbf{x}) \leq f_{d}\left(\boldsymbol{I}_{C r}(\mathbf{x})\right),
\end{aligned}
$$

where $Y_{\text {Mean }}, C b_{\text {Mean }}$ and $C r_{\text {Mean }}$ are the means of corresponding channels values. The authors chose $\tau=40 . f u(),. f l($.$) and f d($.$) are three polynomial functions corresponding to the$ limits of an area formed by the values of fire pixels in the $C r-C b$ color plane. This area is computed from 1000 fire images.

\subsubsection{Proposed algorithm: Bayesian segmentation in $Y C_{b} C_{r}$ color space}

Bayesian fire detection employs a learning based fire pixel detection. Samples of fire and non-fire images are collected from the Internet. 500 natural image from Berkeley dataset [23] are used for non-fire pixel representation. Meanwhile, 400 images which are manually labeled are used for fire pixel representation. In order to reduce the illumination effects and to use only the color information, $Y C_{b} C_{r}$ color space is employed. The $Y$ component is ignored as it changes with the degree of available illumination on the observed scene. The chrominance components $C_{b}$ and $C_{r}$ are employed to represent fire and non-fire pixel information. Let the 
measurement vector be $\mathbf{M}(\mathbf{x})=\left(C_{b}(\mathbf{x}), C_{r}(\mathbf{x})\right)$ for a pixel $(\mathbf{x})$, Bayes' theorem is used to compute the conditional probabilities:

$$
P(\text { fire } \mid \mathbf{M}(\mathbf{x}))=\frac{P(\mathbf{M}(\mathbf{x}) \mid \text { fire }) P(\text { fire })}{P(\mathbf{M}(\mathbf{x}))}
$$

and

$$
P(\text { non-fire } \mid \mathbf{M}(\mathbf{x}))=\frac{P(\mathbf{M}(\mathbf{x}) \mid \text { nonfire }) P(\text { non-fire })}{P(\mathbf{M}(\mathbf{x}))}
$$

Using the conditional probabilities, a pixel $\mathbf{x}$ is classified as fire if the following condition is satisfied

$$
P(\text { fire } \mid \mathbf{M}(\mathbf{x}))>P(\text { non-fire } \mid \mathbf{M}(\mathbf{x}))
$$

which implies that

$$
\frac{P(\mathbf{M}(\mathbf{x}) \mid \text { fire }) P(\text { fire })}{P(\mathbf{M}(\mathbf{x}))}>\frac{P(\mathbf{M}(\mathbf{x}) \mid \text { nonfire }) P(\text { non-fire })}{P(\mathbf{M}(\mathbf{x}))}
$$

and

$$
P(\mathbf{M}(\mathbf{x}) \mid \text { fire }) P(\text { fire })>P(\mathbf{M}(\mathbf{x}) \mid \text { nonfire }) P(\text { non-fire })
$$

It is difficult to estimate $P$ (fire) and $P$ (non-fire), however, the ratio of them $\tau=P$ (fire) $/ P$ (non-fire) is used as a design parameter, i.e.,

$$
\frac{P(\mathbf{M}(\mathbf{x}) \mid \text { fire })}{P(\mathbf{M}(\mathbf{x}) \mid \text { nonfire })}>\frac{P(\text { non-fire })}{P(\text { fire })}=\tau
$$

where $\tau=1$, which states that $P$ (fire) $=P$ (non-fire), is used. The conditional probabilities $P(\mathbf{M}(\mathbf{x}) \mid$ fire $)$ and $P(\mathbf{M}(\mathbf{x}) \mid$ non-fire $)$ are estimated from $2 \mathrm{D}$ histograms of $C_{b}$ and $C_{r}$ color channels. The fire and non-fire images of the training dataset are used to create $2 \mathrm{D}$ histograms of $C_{b}$ and $C_{r}$ color channels to create a representation for fire and non-fire pixels. 
The proposed Bayesian segmentation is a generic representation. It allows different representations for the conditional probabilities, e.g, the measurement vector can be created from features such as texture, gradient information and combinations of color spaces and multivariate Gaussian distribution, and can be used as generative model for conditional probability representation of fire and non-fire pixels. The system's performance can be improved by changing value of the parameter $\tau$ which is a ration of a priori probabilities for fire and non-fire classes. This value can be adapted according to the environment conditions.

\subsection{HSI color space segmentation}

Horng et al. in [16] proposed a method based on the HSI color space. Thresholds are used on each channel of HSI color space for the segmentation. The validity of three conditions are checked for darker environments, i.e.,

$$
\begin{aligned}
& 0^{\circ} \leq \boldsymbol{I}_{H}(\mathbf{x}) \leq 60^{\circ} \\
& 20 \leq \boldsymbol{I}_{S}(\mathbf{x}) \leq 100 \\
& 100 \leq \boldsymbol{I}_{I}(\mathbf{x}) \leq 255
\end{aligned}
$$

and for brighter environments, i.e.,

$$
\begin{aligned}
& 0^{\circ} \leq \boldsymbol{I}_{H}(\mathbf{x}) \leq 60^{\circ} \\
& 40 \leq \boldsymbol{I}_{S}(\mathbf{x}) \leq 100 \\
& 127 \leq \boldsymbol{I}_{I}(\mathbf{x}) \leq 255
\end{aligned}
$$

The thresholds are chosen by the authors by an empirical analysis of 70 flame images. 


\section{$2.4 L^{*} a^{*} b^{*}$ color space segmentation}

Chitade and Katiyar [20] have proposed a color segmentation for satellite image segmentation. Rudz et al. [13] proposed to use this method for fire detection applications. A K-means clustering is applied on the $a^{*}$ and $b^{*}$ channels with $\mathrm{k}$ clusters. The clusters are sorted according to the mean of the $a^{*}$ and $b^{*}$ channels value of their centroid. For the fire segmentation a number of 4 clusters is chosen and only the pixels of the cluster with the highest mean are labeled as fire.

Celik [15] proposed a set of rules on the CIE $L^{*} a^{*} b^{*}$ color space to segment a fire. The following rules must be satisfied to label the pixel $(\mathbf{x})$ as a fire pixel.

$$
\begin{aligned}
& \boldsymbol{I}_{L^{*}}(\mathbf{x}) \geq L_{\text {Mean }}^{*} ; \\
& \boldsymbol{I}_{a^{*}}(\mathbf{x}) \geq a_{\text {Mean }}^{*} ; \\
& \boldsymbol{I}_{b^{*}}(\mathbf{x}) \geq b_{\text {Mean }}^{*} ; \\
& \boldsymbol{I}_{b^{*}}(\mathbf{x}) \geq \boldsymbol{I}_{a^{*}}(\mathbf{x}) ; \\
& P\left(\boldsymbol{I}_{L^{*}}(\mathbf{x}), \boldsymbol{I}_{a^{*}}(\mathbf{x}), \boldsymbol{I}_{b^{*}}(\mathbf{x})\right) \geq \tau .
\end{aligned}
$$

where $L_{\text {Mean }}^{*}, a_{\text {Mean }}^{*}$ and $b_{\text {Mean }}^{*}$ are the mean values of $L^{*}, a^{*}$ and $b^{*}$ color channels, respectively, and $P\left(\boldsymbol{I}_{L^{*}}(\mathbf{x}), \boldsymbol{I}_{a^{*}}(\mathbf{x}), \boldsymbol{I}_{b^{*}}(\mathbf{x})\right)$ is a joint probability that $\left(\boldsymbol{I}_{L^{*}}(\mathbf{x}), \boldsymbol{I}_{a^{*}}(\mathbf{x})\right.$, $\left.\boldsymbol{I}_{b^{*}}(\mathbf{x})\right)$ is a fire pixel, and $\tau$ is a threshold. To compute the joint probability, the author studied the correlation between $L^{*}, a^{*}$, and $b^{*}$ values of fire pixels using the histogram of fire pixels. 500 manually labeled fire pixels are used to compute histograms of the 3 color planes $\left(L^{*}-a^{*}\right),\left(L^{*}-b^{*}\right)$ and $\left(a^{*}-b^{*}\right)$ where channels $L^{*}, a^{*}$, and $b^{*}$ are quantized into 24 levels. From each histogram it is deduced the probability of a color to be a fire color according to this value in the two corresponding channels. $\tau$ is a threshold fixed to 0.005 using receiver operating characteristic (ROC) analysis on the dataset. 


\subsection{Hybrid color space segmentation}

Chen et al. [18] have developed a fast segmentation procedure that was used for fire detection [24]. The method is based on $R, G, B$ color channels and saturation $(S)$ values. Three conditions are checked to classify a pixel (x) as a fire pixel:

$$
\begin{aligned}
& \boldsymbol{I}_{R}(\mathbf{x}) \geq \boldsymbol{I}_{G}(\mathbf{x})>\boldsymbol{I}_{B}(\mathbf{x}) \\
& \boldsymbol{I}_{R}(\mathbf{x})>R_{T} \\
& \boldsymbol{I}_{S}(\mathbf{x}) \geq\left(255-\boldsymbol{I}_{R}(\mathbf{x})\right) \frac{S_{T}}{R_{T}} .
\end{aligned}
$$

where $R_{T}$ is a threshold on the $R$ channel which must be between 115 to 135 and $S_{T}$ is a threshold on the saturation which must be between 55 to 65 .

Rossi and Akhloufi [19] use the channel $V$ of $Y U V$ system for segmenting the fire. They used the K-means algorithm on the channel $V$ to extract the regions corresponding to fire. Here the number of classes $k$ is 2 in order to differentiate fire regions from the background. Considering the fact that the fire occupies the main extracted area from the channel V, the authors propose a learning of $R G B$ values of the image area to establish a reference model for pixels classification. They propose the use of a 3D Gaussian model. Thus, the segmentation condition is:

$$
\sqrt{\left(\boldsymbol{I}_{R}(\mathbf{x})-R_{\text {mean }}\right)^{2}+\left(\boldsymbol{I}_{G}(\mathbf{x})-G_{\text {mean }}\right)^{2}+\left(\boldsymbol{I}_{B}(\mathbf{x})-B_{\text {mean }}\right)^{2}} \leq t \sigma
$$

With $t$ a constant, $R_{\text {mean }}, G_{\text {mean }}, B_{\text {mean }}$ means of extracted area's channels and $\sigma=\max \left(\sigma_{R}, \sigma_{G}, \sigma_{B}\right)$ with $\sigma_{c}$ the standard deviation of channel $c$ of extracted area.

$C_{b}$ channel of $Y C_{b} C_{r}$ color space was chosen by Rudz et al. [13] in order to apply a 
K-means clustering on the image. After this clustering, an improvement is made on the $R G B$ color space according to the size of the cluster to eliminate false pixels. For a large size of a cluster, the following set of rules are used to detect the fire pixels, i.e.,

$$
\begin{aligned}
& \| \text { hist }_{R}^{\text {ref }}-\operatorname{hist}_{R} \|<\tau_{R} ; \\
& \| \text { hist }_{G}^{\text {ref }}-\operatorname{hist}_{G} \|<\tau_{G} ; \\
& \| \text { hist }_{B}^{\text {ref }}-\operatorname{hist}_{B} \|<\tau_{B} .
\end{aligned}
$$

where hist ${ }_{c}^{r e f}$, hist $c$ and $\tau_{c}$ are respectively the reference histogram, the histogram of the candidate pixels and the threshold in color channel $c$. Then, for a small size of a cluster, the following rule set is used to detect the fire pixels:

$$
\begin{aligned}
& \left\|\mu_{R}^{r e f}-\mu_{R}\right\|<\rho_{R} \sigma_{R}^{r e f} ; \\
& \left\|\mu_{G}^{r e f}-\mu_{G}\right\|<\rho_{G} \sigma_{G}^{r e f} ; \\
& \left\|\mu_{B}^{r e f}-\mu_{B}\right\|<\rho_{B} \sigma_{B}^{r e f} .
\end{aligned}
$$

where $\mu_{c}^{r e f}$ and $\sigma_{c}^{r e f}$ are, respectively, the reference mean and the reference standard deviation of fire pixels in the color channel $c, \mu_{c}$ is the mean of candidate fire pixels in the color channel $c$, and $\rho_{c}$ is a coefficient in the color channel $c$. In [13], hist $t_{c}^{r e f}, \mu_{c}^{r e f}$ and $\sigma_{c}^{r e f}$ are computed on the third of the images of the dataset, randomly selected. The thresholds $\tau_{R}, \tau_{G}, \tau_{B}, \rho_{R}, \rho_{G}$ and $\rho_{B}$ are optimized to have the best segmentations on the same part of the dataset according to the F-score (see section 3) using a direct pattern search method [25].

\section{Evaluation criteria}

In the field of image segmentation, there is few works on performance evaluation [26]. In order to compare the performances of the segmentation methods, we can use standard metrics that 
compare the segmented image to a manually segmented image (the ground truth). In this study, 4 metrics are used to compare the color segmentation methods described in section 2. All these metrics are normalized so that the values are between 0 and 1 with the score 1 representing a perfect segmentation.

\subsection{Matthews Correlation Coefficient $(M C C)$}

Proposed by Matthews in [27] for a biochemical evaluation application, this metric is also used for image segmentation evaluation, and more specifically for fire segmentation evaluation [11]. The Matthew correlation coefficient $(M C C)$ is the geometric mean of the regression coefficient and its dual. It is defined as follow:

$$
M C C=\frac{(T P * T N)-(F P * F N)}{\sqrt{(T N+F N)(T N+F P)(T P+F N)(T P+F P)}}
$$

where $T P, T N, F P$ and $F N$ are respectively the number of true positives (true in the segmentation and the ground truth), true negatives (false in the segmentation and the ground truth), false positives (true in the segmentation and false in the ground truth) and false negatives (false in the segmentation and true in the ground truth).

\section{$3.2 \quad$ F1-Score $(F 1)$}

This metric (also named F-score) is mostly used in information retrieval [28] but has also applications in image segmentation evaluation [11]. It involves two measures called precision $(\operatorname{Pr})$ and recall $(R e)$ defined as follow:

$$
\operatorname{Pr}=\frac{T P}{T P+F P}, R e=\frac{T P}{T P+F N}
$$

The F1-Score $(F 1)$ is the harmonic mean of precision and recall: 


$$
F 1=2 * \frac{\operatorname{Pr} * \operatorname{Re}}{\operatorname{Pr}+R e}
$$

\subsection{Hafiane quality index $(H A F)$}

This criterion has been developed for fire segmentation evaluation [29]. It takes into account the position, shape and size of the segmented regions together with the under or over segmentation. First a matching index is defined as follow:

$$
M=\frac{1}{\operatorname{Card}\left(\boldsymbol{I}^{S}\right)} \sum_{j=1}^{N R_{s}} \frac{\operatorname{Card}\left(R_{i^{*}}^{G T} \cap R_{j}^{S}\right) \times \operatorname{Card}\left(R_{j}^{S}\right)}{\operatorname{Card}\left(R_{i^{*}}^{G T} \cup R_{j}^{S}\right)}
$$

where $N R^{S}$ is the number of connected regions in the segmentation result $\boldsymbol{I}^{S} . R_{j}^{S}$ is one of these regions and $R_{i^{*}}^{G T}$ is the region in the reference image $\boldsymbol{I}^{G T}$ that has the most important overlapping surface with the $R_{j}^{S}$ region. Then, in order to take into account the under or over segmentation, another index is defined:

$$
\eta= \begin{cases}N R^{G T} / N R^{S} & \text { if } N R^{S} \geq N R^{G T} \\ \log \left(1+N R^{S} / N R^{G T}\right) & \text { otherwise }\end{cases}
$$

The Hafiane $(H A F)$ quality index is given by the following equation:

$$
H A F=\frac{M+m \times \eta}{1+m}
$$

where $m$ is a weighting factor set to 0.5 .

\section{Image dataset}

In order to benchmark algorithms and/or to test new ones, it is important to work with databases that have a large number of characterized data. Publicly available databases are 
particularly useful to research communities. For example the Bilkent University fire clip dataset [30] provides fire and smoke video clips. Dyntex [31] is another one which provides a diverse collection of high-quality texture videos. For each sequence, an XML-description characterizing both its content, as well as the context in which it was recorded is available. Wildland fire segmentation research is still young and so far no database suitable for this research is available. In this paper, an image dataset of characterized outdoor vegetation fire images is presented. For now, it is not downloadable for author right reason but a website that allows to test algorithms on it is available. Octave/Matlab code are accepted as input and produces benchmarking scores on the dataset. The website can be reached at http://firetest.cs.wits.ac.za/benchmark/.

\subsection{Images of the dataset}

The image dataset used in this work contains 100 RGB images of outdoor vegetation fires at different sizes (from 333x500 pixels to 2592x1944 pixels) and different image formats (jpeg, ppm, bmp). Images are from Internet, others were acquired by the researchers of the UMR CNRS 6134 SPE - University of Corsica and theirs partners (researchers, forester and firefighters) during experiments, controlled burns and wildland fires. The images are pictures of outdoor vegetation fires in which the fire areas are easily segmented by a human eye. Images were taken in different places (Portugal, United States of America, Africa and French regions : Corsica, French riviera, Landes), with different environment (forest, maquis shrubland, rocks, snow,...) and luminosity characteristics (sunny, cloudy, gray sky, blue sky, night, day,...). Example of the dataset images can be seen in the Figure 1.

Each image has been manually segmented by an expert. This manual segmentation is called ground truth and noted $\boldsymbol{I}^{G T}$ where $\boldsymbol{I}^{G T}(\mathbf{x})=1$ if $\mathbf{x}$ is a fire pixel and $\boldsymbol{I}^{G T}(\mathbf{x})=0$ otherwise. Figure 2 presents a fire image with its ground truth. 

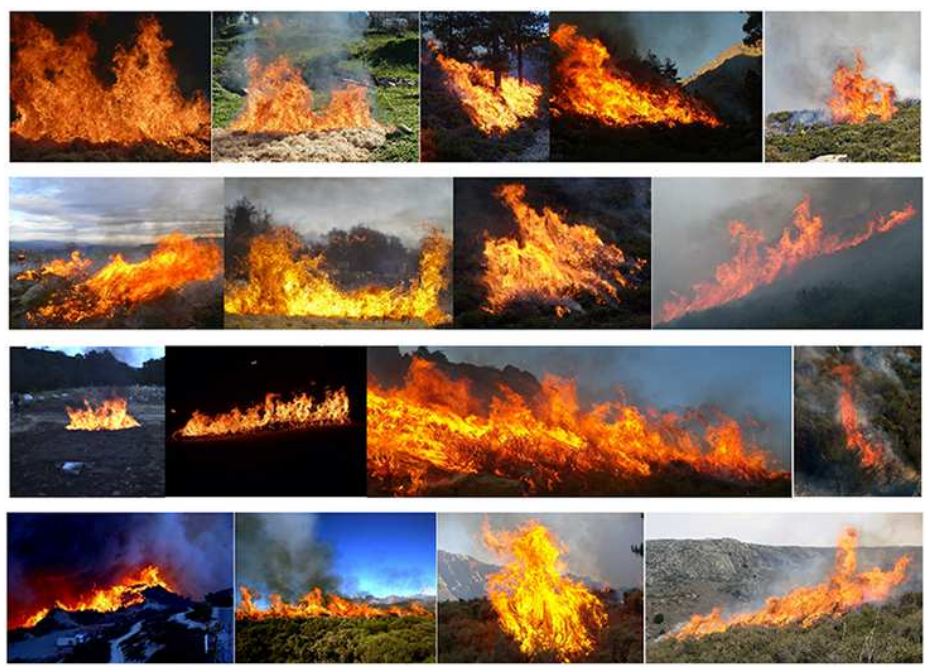

Figure 1: Example of the dataset images. Fires are in different locations, with different fuels and different luminosity conditions.

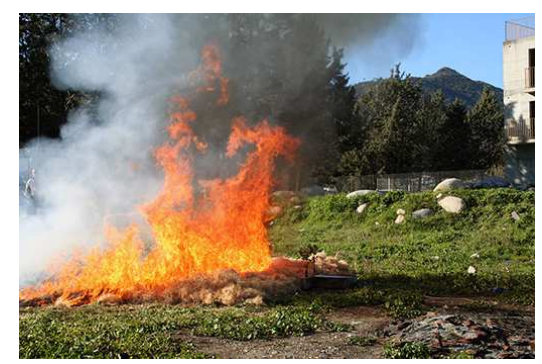

$(a)$

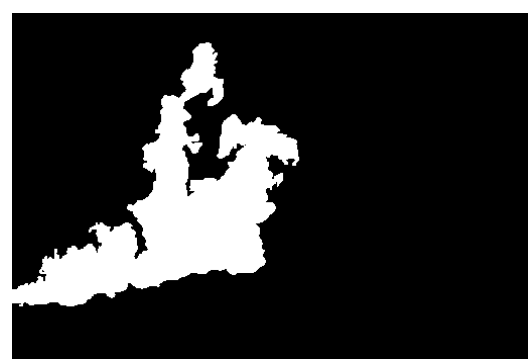

(b)

Figure 2: Original image $(a)$ and its ground truth $(b)$

\subsection{Image characterization}

In each image of the dataset, the fire areas are used to characterize:

- the percentage of fire pixels present in the image;

- the dominant color of the fire;

- the presence of smoke; 
- the light intensity of the environment.

Using these criteria, the images are then automatically classified. An example of this categorization is shown in figure 3 .

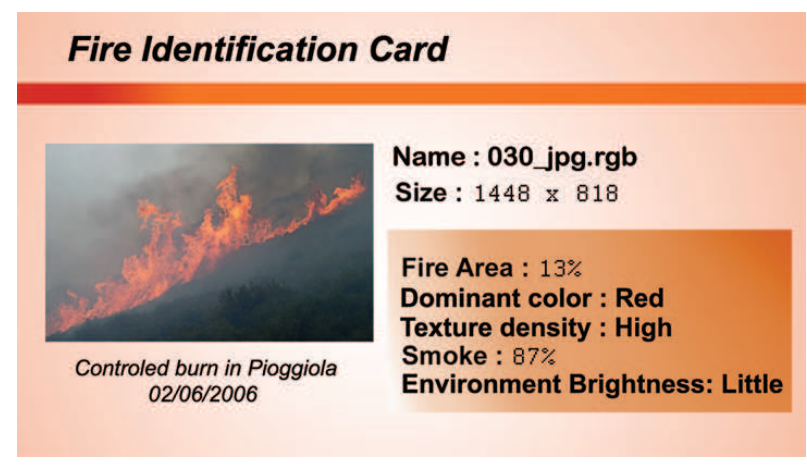

Figure 3: Fire ID

\subsubsection{Color}

In Zhao et al. [32], the authors propose to label fire pixels in three types of colors: red, orange and white-yellow. In our work, pixels are labeled to one of these colors using the $H S L$ color space. The $S$ channel gives information about color saturation of the pixel. If the saturation is low, the pixel have no color (gray), so the first condition to verify for all the fire pixels is:

$$
\boldsymbol{I}_{S}(\mathbf{x}) \geq 50
$$

If equation (24) is not satisfied, the pixel $\mathbf{x}$ is labeled as "other color". Else, if one of the two following conditions are satisfied, the pixel is labeled as white-yellow:

$$
\boldsymbol{I}_{V}(\mathbf{x}) \geq 200
$$




$$
42^{\circ}<\boldsymbol{I}_{H}(\mathbf{x}) \leq 64^{\circ}
$$

Where equation (25) corresponds to white colors and equation (26) corresponds to yellow colors. Fire pixels are labeled as orange if equation (25) is false and if the following condition is satisfied:

$$
14^{\circ}<\boldsymbol{I}_{H}(\mathbf{x}) \leq 42^{\circ}
$$

And fire pixels are labeled red if equation (25) is false and if:

$$
-57^{\circ}<\boldsymbol{I}_{H}(\mathbf{x}) \leq 14^{\circ}
$$

If none of these conditions are satisfied the pixel is labeled as "other color". The thresholds used in these conditions are defined empirically from our experiments and from the hue values of the shade of colors [33].

For example, the fire in Figure $4(a)$ is divided in 3 different colors in Figure $4(b),(c)$ and $(d)$. To classify the color of the fire in each image, the predominant color is found with the color ratio of fire pixels. As a majority of pixels are orange for the fire presented in Figure 4, the fire is classified as orange.

\subsubsection{Smoke}

Smoke is the principal problem in color segmentation of fires. If dense smoke masks part of the fire, it is impossible without other information to segment the hidden part. The image dataset does not contain dense smoke images but images with thin smoke that are interesting for wildland fire segmentation are present. To determine if there is slight smoke in front of the fire, a learning has been done on fire pixels with and without smoke. Pixels of the images are classified as "smoke" or "smokeless" using Support Vector Machines. If there is more than $1 / 2$ of fire pixels which are classified as "smoke" then the image is classified as "with smoke" 


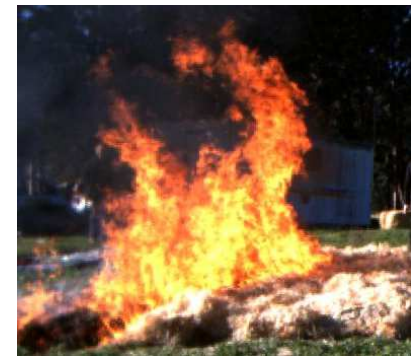

$(a)$

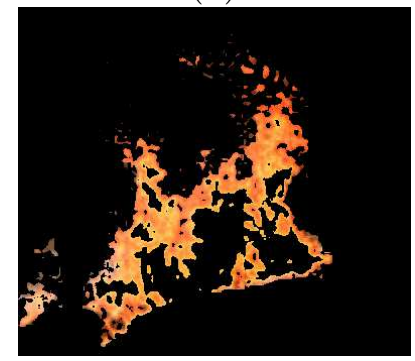

$(c)$

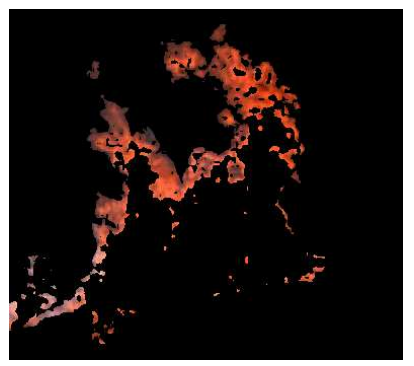

(b)

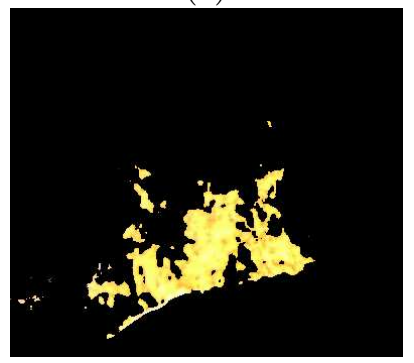

$(d)$

Figure 4: Decomposition of color in a fire image: $(a)$ fire image, $(b)$ red pixels, $(c)$ orange pixels, and $(d)$ white-yellow pixels. This fire is composed of $31 \%$ of red pixels, $39 \%$ of orange pixels and $25 \%$ of withe-yellow pixels.

(see Figure 5).

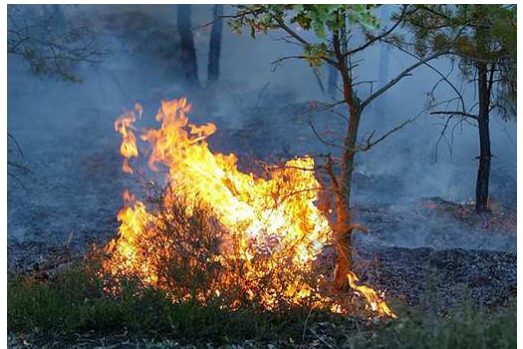

$(a)$

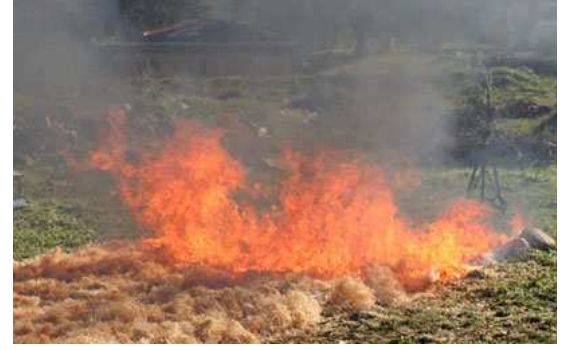

(b)

Figure 5: Examples of characterizations. (a) "smokeless" fire and (b) fire with smoke 


\subsubsection{Brightness of the environment}

The scene can be characterized by the brightness of the environment. To estimate it, an average of the channel I of HSI is computed using the background of the image. The considered background is the complementary of the fire in the image (i.e. $\mathbf{x} \in \boldsymbol{I} / G T(\mathbf{x})=0)$. The image is classified according to the value of this mean. Two thresholds are chosen empirically from our experiments in order to classify the background brightness: $\tau^{h}=45$ and $\tau^{l}=20$. The environment is classified as "high intensity", if the mean of the intensity of the background is upper than $\tau^{h}$. It is classified as "medium intensity", if the mean of intensity is between $\tau^{h}$ and $\tau^{l}$. It is classified as "low intensity" if the intensity is lower than $\tau^{l}$. (see Figure 6).

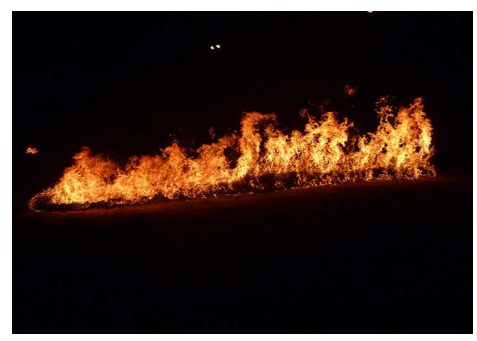

(a)

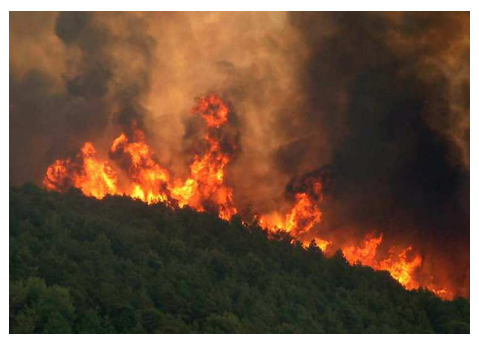

(b)

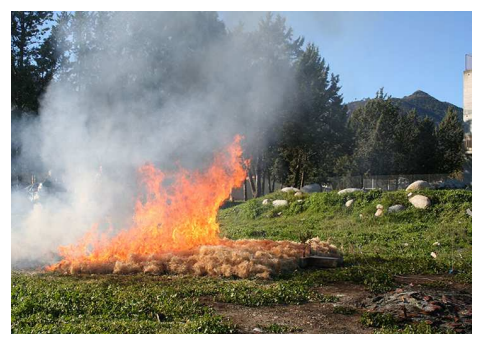

$(c)$

Figure 6: Environment classified with low intensity $(a)$, medium intensity $(b)$ high intensity (c)

These categorizations will help to have a more precise evaluation of the efficiency of the color segmentation algorithms.

The images of the dataset proposed in this article were classified using the above criteria. Table 2 presents the results of this classification.

Table 2: Number of images of the dataset per category

\begin{tabular}{|c|c|c|c|c|c|c|c|c|}
\hline \multicolumn{3}{|c|}{ Fire color } & \multicolumn{2}{|c|}{ Smoke } & \multicolumn{3}{|c|}{$\begin{array}{l}\text { Environment } \\
\text { intensity }\end{array}$} & \multirow[t]{2}{*}{ Total } \\
\hline Red & Orange & White & With & Without & Low & medium & High & \\
\hline 23 & 75 & 2 & 58 & 42 & 48 & 56 & 30 & 100 \\
\hline
\end{tabular}




\section{Benchmarking}

The 12 fire color segmentation algorithms have been tested on the wildland fire image dataset. For more consistency in the segmentation, the same post processing is applied on each method. The post processing keeps the largest regions and fills these regions in order to remove false positive pixels of the segmentation. The post processing improves segmentation results without penalize the benchmarking process.

Some of the presented methods need to compute a threshold. Rudz et al. [13] propose to compute these thresholds from a third of the images of the dataset taken randomly. The value of the threshold that maximizes the F-score for these images is estimated with a direct pattern search algorithm [25]. In this study, 33 images were chosen randomly from the dataset keeping the same ratio for each category. This part of the dataset will be named the training dataset.

The direct pattern search on the training dataset has been used to find thresholds for the following methods: Phillips et al., Chen et al. Ko, Celik $L^{*} a^{*} b^{*}$ and Rudz et al.. For the dataset proposed we obtain $\tau=-1.78 \times 10^{-3}$ for Phillips method. For the color segmentation method of Chen et al. [18] an analysis of the best value for $R_{T}$ and $S_{T}$ thresholds has been done. The result of the direct pattern search gives $R_{T}=115$ and $S_{T}=55$ as the best thresholds. A change of these values on the intervals proposed in [18] doesn't impact much the segmentation. Indeed the standard deviation of the false positive rate is $2 \times 10^{-4}$ on average and $2 \times 10^{-3}$ for the standard deviation of true positive rate for the 231 combinations of $R_{T}$ and $S_{T}$. Thresholds of $1.46 \times 10^{-5}$ for Ko's method [10] and 0.02453 for Celik's method

[15] have been found. For the color segmentation of Rudz [13], hist ${ }_{R}^{\text {ref }}$, hist ${ }_{G}^{\text {ref }}$ and $h i s t_{B}^{\text {ref }}$ were normalized in $[0,1]$. The following thresholds were found: $\tau_{R}=3.04, \tau_{G}=8.99, \tau_{B}=$ $9, \rho_{R}=0.75, \rho_{G}=7$ and $\rho_{B}=3$. Values of $\tau_{C}$ are much smaller than the one proposed in the reference article because of the histogram normalization. 
The training dataset has been used for all the methods that need a set of training images (i.e. Phillips et al., Ko et al., Celik $\left(L^{*} a^{*} b^{*}\right)$, Collumeau et al. and Rudz et al.). The color distribution of fire pixels on the 3 different color planes for Celik $L^{*} a^{*} b^{*}$, computed on the training dataset, is shown in Figure 7 .

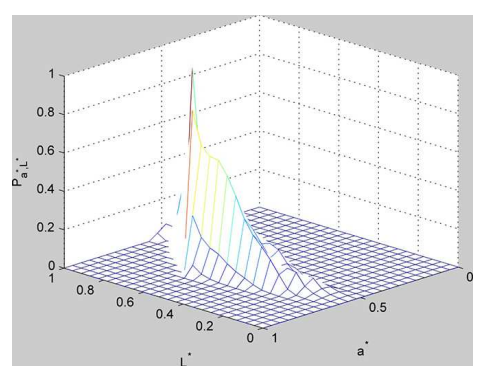

(a)

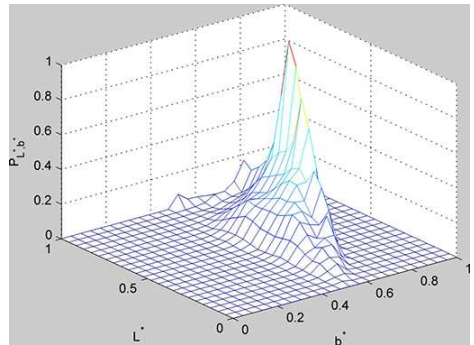

(b)

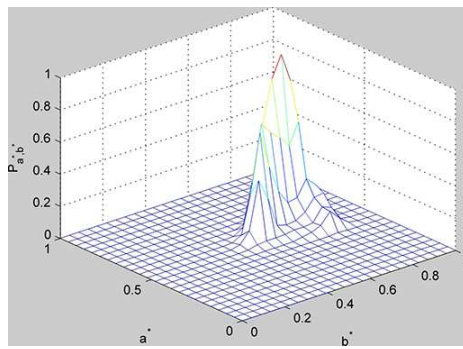

$(c)$

Figure 7: Color distribution of fire pixels on $\left(L^{*}, a^{*}\right)$ color plane $(a),\left(L^{*}, b^{*}\right)$ color plane $(b)$ and $\left(a^{*}, b^{*}\right)$ color plane $(c)$

The fire areas segmented on four images of our dataset by the 12 methods presented in this article are shown in Figure 8. It can be seen that depending of the color of the fire, the fuel, the presence of smoke, the environement, the performance of the methods varies. The twelve color segmentation methods were evaluated on the 100 images of the dataset using the four evaluation scores presented in section 3. Table 3 presents the results according to the fire color, the presence of smoke, and the environment. The two tables are organized as follow: the first ten lines correspond to characteristics presented in section 4.2. "Red", "Orange" and "White" are the dominant color of the fire, "Smoke" indicates if smoke represents more than $10 \%$ of the fire pixels. Three levels are used to characterize the intensity: low, medium and high. The last line corresponds to the average of scores for the 100 images of the dataset. For each line, best scores of the twelve methods are underlined. For the dataset presented in this work, the methods of Phillips et al. and Collumeau et al. give the bests results. For these methods, the categories that obtain highest scores correspond to the categories with more 
images in the training dataset ("orange", "medium intensity"). This is explained by the high importance of the learning step in both methods. Indeed as the training dataset contains few images with withe-yellow pixels, the images of fire with a majority of white-yellow pixel obtain lower scores than for orange fire. The methods of Rossi and Akhloufi, Chitade and Katiyar and the Bayesian color segmentation give good results and can be used for techniques without prior learning. Results show that Rossi and Akhloufi segmentation is more robust to smoke than the others methods. The method of Chen gives the best results for white-yellow fires but has lower score for orange or red fires. The environment characterization shows that this method works better with dark environments. So the Chen segmentation should be used on night fire images. It can be noted that methods on the $Y_{C} b C_{r}$ color space are also more efficient on night fire as their scores are better for dark environment and for white-yellow fires. 
Table 3: Scores of fire segmentation methods

\begin{tabular}{|c|c|c|c|c|c|c|c|c|c|}
\hline & \multicolumn{3}{|c|}{ Phillips et al. [7] } & \multicolumn{3}{|c|}{ Chen et al. [18] } & \multicolumn{3}{|c|}{ Horng et al. [16] } \\
\hline & $\mathrm{MCC}$ & F1 & $\mathrm{HAF}$ & $\mathrm{MCC}$ & F1 & $\mathrm{HAF}$ & $\mathrm{MCC}$ & $\mathrm{F} 1$ & $\mathrm{HAF}$ \\
\hline Red & $\underline{0.78}$ & $\underline{0.79}$ & 0.69 & 0.57 & 0.57 & 0.50 & 0.33 & 0.32 & 0.37 \\
\hline Orange & $\underline{0.82}$ & $\underline{0.83}$ & $\underline{0.76}$ & 0.59 & 0.61 & 0.58 & 0.11 & 0.15 & 0.21 \\
\hline White & $\overline{0.60}$ & $\overline{0.59}$ & $\overline{0.62}$ & $\underline{0.75}$ & $\underline{0.75}$ & $\underline{0.73}$ & 0.67 & 0.64 & 0.52 \\
\hline Smoke & $\underline{0.81}$ & $\underline{0.82}$ & $\underline{0.74}$ & 0.56 & 0.58 & 0.55 & 0.19 & 0.22 & 0.28 \\
\hline Smokeless & 0.81 & 0.81 & 0.79 & 0.73 & 0.73 & 0.68 & 0.07 & 0.06 & 0.10 \\
\hline Very low I & $\underline{0.75}$ & $\underline{0.76}$ & $\underline{0.74}$ & $\underline{0.75}$ & 0.75 & 0.73 & 0.09 & 0.04 & 0.07 \\
\hline Medium I & $\overline{0.82}$ & $\overline{0.82}$ & $\overline{0.74}$ & $\overline{0.60}$ & 0.61 & 0.57 & 0.14 & 0.17 & 0.26 \\
\hline High I & $\overline{0.82}$ & $\overline{0.83}$ & $\overline{0.75}$ & 0.49 & 0.52 & 0.49 & 0.26 & 0.32 & 0.33 \\
\hline \multirow[t]{3}{*}{ Average } & $\underline{0.81}$ & $\underline{0.82}$ & $\underline{0.75}$ & 0.59 & 0.60 & 0.57 & 0.17 & 0.20 & 0.25 \\
\hline & \multicolumn{3}{|c|}{ Celik et al. [9] } & \multicolumn{3}{|c|}{ Celik and Demirel [12] } & \multicolumn{3}{|c|}{ Ko et al. [10] } \\
\hline & $\mathrm{MCC}$ & F1 & $\mathrm{HAF}$ & $\mathrm{MCC}$ & $\mathrm{F} 1$ & $\mathrm{HAF}$ & $\mathrm{MCC}$ & $\mathrm{F} 1$ & HAF \\
\hline Red & 0.29 & 0.24 & 0.21 & 0.56 & 0.54 & 0.42 & 0.55 & 0.53 & 0.42 \\
\hline Orange & 0.47 & 0.44 & 0.33 & 0.62 & 0.64 & 0.56 & 0.46 & 0.44 & 0.36 \\
\hline White & 0.30 & 0.27 & 0.28 & 0.63 & 0.66 & 0.53 & 0.25 & 0.29 & 0.34 \\
\hline Smoke & 0.44 & 0.39 & 0.30 & 0.59 & 0.60 & 0.51 & 0.48 & 0.46 & 0.36 \\
\hline Smokeless & 0.39 & 0.35 & 0.26 & 0.73 & 0.74 & 0.64 & 0.47 & 0.47 & 0.41 \\
\hline Very low I & 0.31 & 0.28 & 0.23 & 0.62 & 0.63 & 0.54 & 0.46 & 0.42 & 0.38 \\
\hline Medium I & 0.45 & 0.42 & 0.32 & 0.64 & 0.66 & 0.57 & 0.51 & 0.48 & 0.33 \\
\hline High I & 0.45 & 0.39 & 0.29 & 0.53 & 0.54 & 0.45 & 0.51 & 0.48 & 0.33 \\
\hline \multirow[t]{3}{*}{ Average } & 0.43 & 0.39 & 0.30 & 0.61 & 0.62 & 0.53 & 0.48 & 0.46 & 0.37 \\
\hline & \multicolumn{3}{|c|}{ Rossi and Akhloufi [19] } & \multicolumn{3}{|c|}{ Chitade and Katiyar [20] } & \multicolumn{3}{|c|}{ Celik [15] } \\
\hline & $\mathrm{MCC}$ & $\mathrm{F} 1$ & HAF & MCC & F1 & $\mathrm{HAF}$ & $\mathrm{MCC}$ & $\mathrm{F} 1$ & $\mathrm{HAF}$ \\
\hline Red & 0.76 & 0.76 & 0.71 & 0.71 & 0.70 & 0.60 & 0.25 & 0.19 & 0.11 \\
\hline Orange & 0.75 & 0.76 & $\overline{0.70}$ & 0.73 & 0.74 & 0.66 & 0.43 & 0.41 & 0.29 \\
\hline White & 0.57 & 0.56 & 0.60 & 0.59 & 0.58 & 0.61 & 0.11 & 0.10 & 0.12 \\
\hline Smoke & 0.75 & 0.76 & 0.70 & 0.72 & 0.72 & 0.63 & 0.37 & 0.33 & 0.25 \\
\hline Smokeless & 0.75 & 0.77 & 0.73 & 0.76 & 0.78 & 0.73 & 0.48 & 0.45 & 0.24 \\
\hline Very low I & 0.69 & 0.73 & 0.71 & 0.73 & 0.75 & 0.73 & 0.39 & 0.36 & 0.20 \\
\hline Medium I & 0.76 & 0.76 & 0.71 & 0.73 & 0.73 & 0.65 & 0.38 & 0.35 & 0.26 \\
\hline High I & 0.76 & 0.77 & 0.69 & 0.70 & 0.70 & 0.60 & 0.39 & 0.34 & 0.25 \\
\hline \multirow[t]{3}{*}{ Average } & 0.75 & 0.76 & 0.70 & 0.72 & 0.73 & 0.64 & 0.38 & 0.35 & 0.25 \\
\hline & \multicolumn{3}{|c|}{ Collumeau et al. [11] } & \multicolumn{3}{|c|}{ Rudz et al. [13] } & \multicolumn{3}{|c|}{ Bayesian segmentation } \\
\hline & $\mathrm{MCC}$ & F1 & $\mathrm{HAF}$ & $\mathrm{MCC}$ & F1 & $\mathrm{HAF}$ & $\mathrm{MCC}$ & $\mathrm{F} 1$ & $\mathrm{HAF}$ \\
\hline Red & 0.74 & 0.73 & 0.66 & 0.53 & 0.49 & 0.43 & 0.66 & 0.64 & 0.53 \\
\hline Orange & 0.79 & 0.80 & 0.75 & 0.59 & 0.58 & 0.53 & 0.69 & 0.71 & 0.64 \\
\hline White & 0.72 & 0.72 & 0.71 & 0.64 & 0.67 & 0.56 & 0.69 & 0.69 & 0.69 \\
\hline Smoke & 0.77 & 0.77 & 0.72 & 0.55 & 0.54 & 0.49 & 0.64 & 0.65 & 0.58 \\
\hline Smokeless & $\underline{0.85}$ & $\underline{0.85}$ & $\underline{0.81}$ & 0.72 & 0.70 & 0.64 & 0.74 & 0.75 & 0.56 \\
\hline Low I & $\overline{0.73}$ & $\overline{0.74}$ & $\overline{0.70}$ & 0.59 & 0.59 & 0.53 & 0.73 & 0.74 & 0.70 \\
\hline Medium I & 0.79 & 0.79 & 0.74 & 0.60 & 0.58 & 0.54 & 0.70 & 0.71 & 0.63 \\
\hline High I & 0.78 & 0.79 & $\overline{0.73}$ & 0.53 & 0.51 & 0.45 & 0.63 & 0.64 & 0.54 \\
\hline Average & 0.78 & 0.78 & 0.73 & 0.58 & 0.56 & 0.51 & 0.69 & 0.69 & 0.61 \\
\hline
\end{tabular}




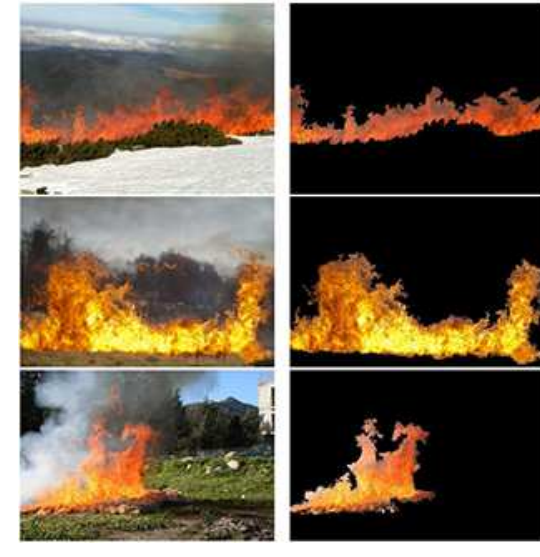

Original image

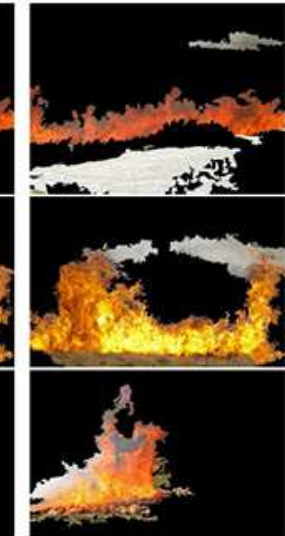

Chen 2004 [18]

ज़

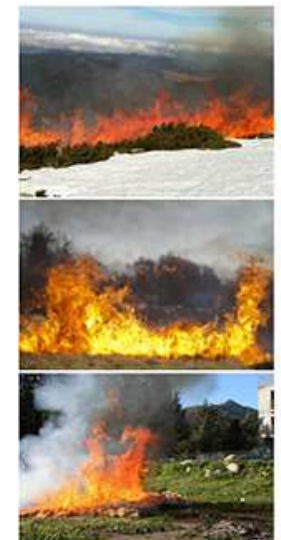

Original image
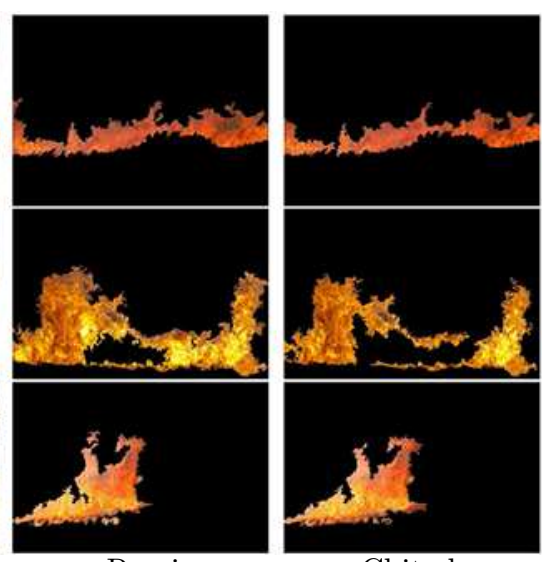

Rossi

2010 [19]

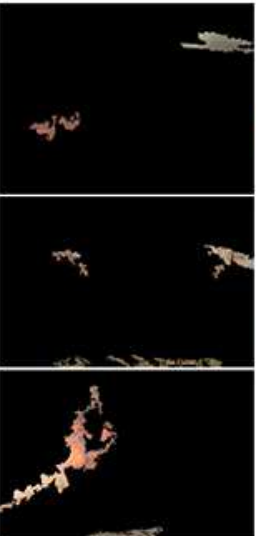

Horng

2005 [16]

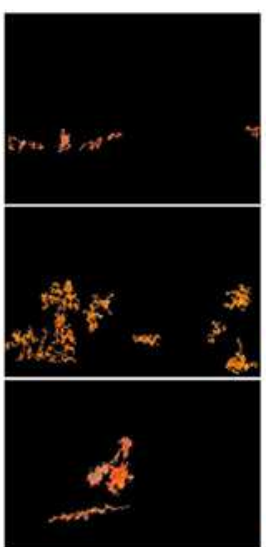

Celik

2010 [15]

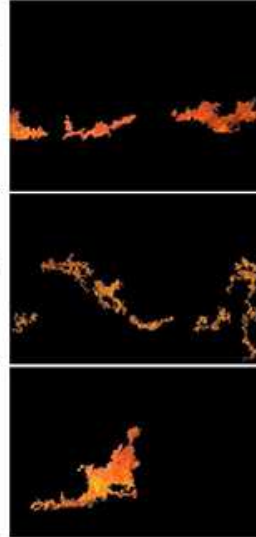

Celik

2007 [9]

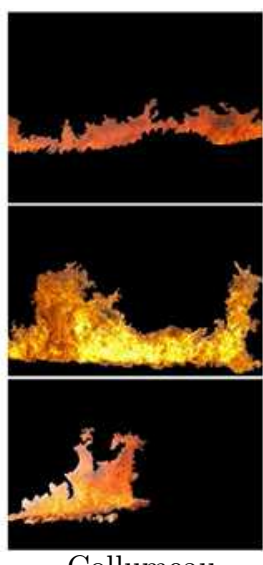

Collumeau

2011 [11]

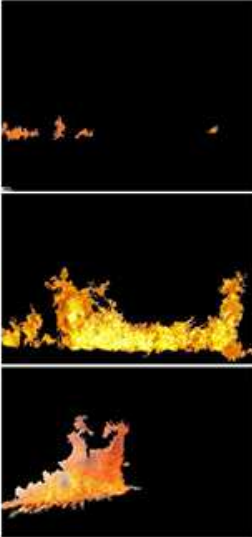

Celik

2009 [12]

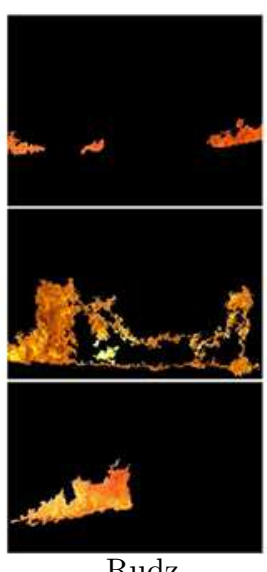

2010 [20]

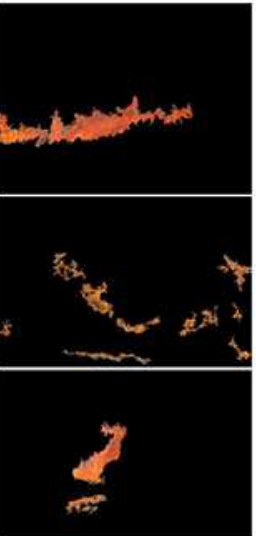

Ko

2009 [10]

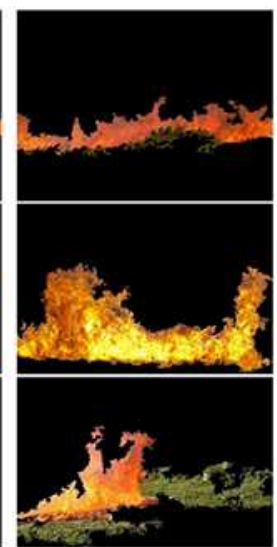

Bayesian

2014

Figure 8: Result of the different methods of fire segmentation on 3 images of the dataset. Segmented images have been multiplied by the $R G B$ images for a better visibility 


\section{Conclusion}

In this work, eleven state of the art algorithms for wildland fire color segmentation were developed and their performances analyzed. Also a new algorithm based on Bayesian conditional probability classification was proposed. Additionally, a new dataset was developed and a new categorization approach was used in order to classify the available images in different meaningful categories. A benchmarking of the twelve algorithms has been made on this dataset using different standard metrics. The obtained results show that the proposed color segmentation techniques performance is dependent on the image category (lighting, predominant color, smoke,...). The Collumeau et al. segmentation is best suited for day fires without smoke. The Rossi and Akhloufi segmentation is robust to smoke, the method of Chitade and Katiyar is robust to environment intensity changes and the Bayesian segmentation is robust to fire color changes. The method of Chen et al. gives best results for white fire images. Since color segmentation is often the first step in a fire segmentation process, this work helps to define the choice of the best algorithm in an operational scenario. Finally, the developed dataset and a benchmarking website were also developed in order to help the research community to compare the performance of their new developed algorithms with the ones presented in this paper in a categorized database. We are currently working on an online database containing a large number of characterized visible and near infrared vegetation fire images and sequences.

\section{References}

[1] 'European forest fire information system', http://forest.jrc.ec.europa.eu/effis/. accessed April 2015.

[2] Grishin, A.M. and Albini, F.A.: 'Mathematical modeling of forest fires and new methods of fighting them' (Publishing house of the Tomsk state university, 1997) 
[3] Balbi, J.H., Rossi, J.L., Marcelli, T., Chatelon, F.J.: 'Physical modeling of surface fire under nonparallel wind and slope conditions', Combustion Science and Technology, 2010, 182, (7), pp 922-939

[4] Santoni, P.A., Simeoni, A., Rossi, J.L., et al.: 'Instrumentation of wildland fire: characterisation of a fire spreading through a Mediterranean shrub', Fire Safety Journal, 2006, 41, (3), pp 171-184

[5] Rothermel, R.C. and Anderson, H.E.: 'Fire spread characteristics determined in the laboratory' (Intermountain Forest and Range Experiment Station, Forest Servcie, US Department of Agriculture, 1966)

[6] Çetin, A.E., Dimitropoulos, K., Gouverneur, B., et al.: 'Video fire detection-review', Digital Signal Processing, 2013, 23, (6), pp 1827-1843

[7] Phillips III, W., Shah, M., da Vitoria Lobo, N.: 'Flame recognition in video', Pattern Recognition Letters, 2002, 23, (1), pp 319-327

[8] Töreyin, B.U., Dedeoğlu, Y., Güdükbay, U., Çetin, A.E.: 'Computer vision based method for real-time fire and flame detection', Pattern Recognition Letters, 2006, 27, (1), pp 4958

[9] Celik, T., Demirel, H., Ozkaramanli, H., Uyguroglu, M.: 'Fire detection using statistical color model in video sequences', Journal of Visual Communication and Image Representation, 2007, 18, (2), pp 176-185, DOI: 10.1016/j.jvcir.2006.12.003

[10] Ko, B.C., Cheong, K.H., Nam, J.Y.: 'Fire detection based on vision sensor and support vector machines', Fire Safety Journal, 2009, 44, (3), pp 322-329 
[11] Collumeau, J.F., Laurent, H., Hafiane, A., Chetehouna, K.: 'Fire scene segmentations for forest fire characterization: A comparative study', Proc. 18th IEEE International Conference on Image Processing (ICIP), Sept. 2011, pp. 2973-2976

[12] Celik, T., Demirel, H.: 'Fire detection in video sequences using a generic color model', Fire Safety Journal, 2009, 44, (2), pp 147-158

[13] Rudz, S., Chetehouna, K., Hafiane, A., Laurent, H., Sero-Guillaume, O.: 'Investigation of a novel image segmentation method dedicated to forest fire applications', Measurement Science and Technology, 2013, 24, (7), pp 075403

[14] Marbach, G., Loepfe, M., Brupbacher, T.: 'An image processing technique for fire detection in video images', Fire safety journal, 2006, 41, (4), pp 285-289

[15] Celik, T.: 'Fast and efficient method for fire detection using image processing', ETRI journal, 2010, 32, (6)

[16] Horng, W.B., Peng, J.W., Chen, C.Y.: 'A new image-based realtime flame detection method using color analysis', Proc. IEEE Networking, Sensing and Control, 2005, pp. 100-105

[17] Liu, C.B., Ahuja, N.: 'Vision based fire detection', Proc. of the 17th International Conference on Pattern Recognition (ICPR), 2004, 4, pp. 134-137

[18] Chen, T.H.,Wu, P.H., Chiou, Y.C.: 'An early fire-detection method based on image processing', Proc. International Conference on Image Processing, (ICIP), 2004, vol. 3, pp. $1707-1710$

[19] Rossi, L., Akhloufi, M., Tison Y.: 'Dynamic fire 3D modeling using a realtime stereovision system', Journal of Communication and Computer, 2010, 6, (10), pp. 54 
[20] Chitade, A.Z. and Katiyar, S.K.: 'Colour based image segmentation using k-means clustering', International Journal of Engineering Science and Technology, 2010, 2, (10), pp. $5319-5325$

[21] Rudz, S., Chetehouna, K., Hafiane, A., Sero-Guillaume, O., Laurent, H.: 'On the evaluation of segmentation methods for wildland fire', Proc. Advanced Concepts for Intelligent Vision Systems, Bordeaux, France, Sept. 2009, pp.12-23

[22] Santana, P., Gomes, P., Barata, J.: 'A vision-based system for early fire detection', Proc. IEEE International Conference on Systems, Man, and Cybernetics (SMC), oct. 2012, pp. $739-744$

[23] Martin, D., Fowlkes, C., Tal, D., Malik, J.: 'A database of human segmented natural images and its application to evaluating segmentation algorithms and measuring ecological statistics', Proc. Eighth IEEE International Conference on Computer Vision (ICCV), 2001, 2, pp.416-423

[24] Habiboğlu, Y.H., Günay, O., Çetin, A.E.: 'Covariance matrix-based fire and flame detection method in video', Machine Vision and Applications, 2011, 23, (6), pp. 1103-1113

[25] Hooke, R., Jeeves, T.A.: 'direct searchsolution of numerical and statistical problems', Journal of the ACM, 1961, 8, (2), pp. 212-229

[26] Jiang, X.: 'Performance evaluation of image segmentation algorithms', Handbook of Pattern Recognition and Computer Vision, 2005, 3, pp. 525-542

[27] Matthews, B.W.: 'Comparison of the predicted and observed secondary structure of T4 phage lysozyme', Biochimica et Biophysica Acta (BBA)-Protein Structure, 1975, 405, (2), pp. $442-451$ 
[28] Van Rijsbergen, C.J.: 'Information Retrieval', (Butterworth-Heinemann, London, 2nd edn. 1979)

[29] Hafiane, A., Chabrier, S., Rosenberger, C., Laurent, H.: 'A new supervised evaluation criterion for region based segmentation methods', Proc. Advanced Concepts for Intelligent Vision Systems, Delft, Netherlands, August 2007, pp. 439-448

[30] 'The Bilkent VisiFire web-page and dataset', http://signal.ee.bilkent.edu.tr/ Visifire, accessed April 2015

[31] Péteri, R., Fazekas, S., Huiskes, M.J.: 'DynTex : a Comprehensive Database of Dynamic Textures',Pattern Recognition Letters, 2010, 31, (12), pp. 1627-1632

[32] Zhao, J., Zhang, Z., Han, S., Qu, C., Yuan, Z., Zhang, D.: 'SVM based forest fire detection using static and dynamic features', Computer Science and Information Systems, 2011, 8, (3), pp. 821-841

[33] 'Work with color', http://www.workwithcolor.com/color-names-01.htm, accessed April 2015 